\title{
Enoxaparin utilization evaluation in inpatients with or at risk of thromboembolic disorders: a one-year, single-centered, retrospective study
}

\author{
Shahram Ala ${ }^{1}$, Gohar Eslami ${ }^{1 *}$, Atieh Sayftabar ${ }^{1}$ \\ ${ }^{1}$ Phramaceutical Resaerch Center, Department of Clinical Pharmacy, Faculty of Pharmacy, Mazandaran University of Medical Sciences, Sari, Iran \\ Received: Mar 14, 2016, Revised: Apr 18, 2016, Accepted: May 6, 2016
}

\begin{abstract}
The aim of this study was evaluation of enoxaparin utilization in hospitalized patients who had received enoxaparin either for prophylaxis or for treatment of thromboembolic disorders. A total of 356 patients were included in this retrospective study and were analyzed for enoxaparin utilization in terms of prescription patterns, treatment strategy, dosing regimen, indications/contraindications, adverse drug reactions, bleeding events, drug-drug interactions and monitoring parameters. The inpatient records and charts were used to extract the relevant data. $47.8 \%$ of the patients were prescribed inappropriate doses of enoxaparin, and $35.7 \%$ were treated with enoxaparin for inappropriate duration. Lack of appropriate monitoring of platelet count, complete blood count (CBC), activated partial thromboplastin time (aPTT), serum potassium level and serum creatinine during enoxaparin therapy was also noted in nearly one third of the patients. Furthermore, dose adjustment was not performed for $85 \%$ of the patients who had elevated serum creatinine. Co-administration of enoxaparin and antiplatelet drugs (aspirin and clopidogrel) or non-steroidal anti-inflammatory drugs were noted in $72 \%$ of the patients. No cases of major bleeding occurred but hematuria was observed in $16.3 \%$ of the patients. High doses of enoxaparin (120 mg/day), concurrent administration of antiplatelet drugs and increased age were found to be the main risk factors for bleeding. Overall, inappropriate utilization of enoxaparin in terms of prescription, dosing regimen, drug interaction and monitoring was observed in a large proportion of the patients which indicates the need for more careful consideration of the patients' conditions and the treatment guidelines before treatment with enoxaparin in the studied healthcare setting.
\end{abstract}

Keywords: Enoxaparine, utilization, evaluation, inappropriate

Pharm Biomed Res 2016; 2(1): 55-65

DOI: 10.18869/acadpub.pbr.2.1.55

\section{Introduction}

Drug utilization evaluation (DUE) is an essential part of pharmacoepidemiology that reviews drug use and prescribing patterns in regard to current recommendations or guidelines for the treatment of a certain disease and assess the extent of inappropriate prescription of drugs as well as the associated clinical, ecological and economic consequences (1). The World Health Organization (WHO) defines drug utilization as "the marketing, 
distribution, prescription and use of drugs in a society, with special emphasis on the resulting medical, social and economic consequences" (2). Inappropriate drug use may result in inadequate treatment, adverse effects, increased cost of medical care and patient mortality. Thus, the principal aim of DUE is to facilitate rational use of drugs, which means prescription of drugs in an optimal dose on the right indication (1).

Enoxaparin is a low molecular weight heparin (LMWH) which is produced by depolymerization of unfractioned heparin (3). It is the leading LMWH with the largest volume of published information on use in the setting of percutaneous coronary intervention (4) and the most widely studied LMWH for the prophylaxis/treatment of venous thromboembolism (VTE) (5). The ease of administration and the predictable anticoagulant effect of enoxaparin which eliminate the need for routine laboratory monitoring (4,6-7), relatively low cost and its wide availability (4) have led to wide applicability and general acceptability of the drug for the prophylaxis/treatment of thromboembolic disorders in most healthcare settings worldwide. Hence, it is a suitable candidate for DUE study. The present DUE study was designed to assess the prescription patterns of enoxaparin in line with current guidelines and its licensed indications, and to identify the adverse effects associated with its inappropriate utilization in hospitalized patients with or at risk of thromboembolic disorders.

\section{Patients and methods}

This retrospective DUE study was carried out in a university-affiliated, general teaching hospital in Babol, Mazandaran, Iran, in a one year period from March 2012 to March 2013. The study was approved by the research ethics committee of Mazandaran University of Medical Sciences The choice of this hospital for the present DUE study was mainly based on the large volume of enoxaparin prescriptions over the past three years, as enoxaparin was the drug of choice for the initial treatment of patients with VT at this hospital. Inpatients from all wards who had received enoxaparin (Clexane ${ }^{\circledR}$, AventisTM) as part of their treatment for at least 24 hours were included in the study. The medical records/ charts of patients were used to extract the following data:

a) The demographic characteristics of the patients

b) Treatment strategies (prophylactic or therapeutic)

c) Indication for use

d) Precautions/contraindications

e) Administered dose and duration of treatment

f) Laboratory monitoring with complete blood count (CBC), platelet count, activated prothrombin time (aPTT), serum creatinin , seum potassium, international normalized ratio (INR), anti Xa 
Factor, urine analysis and stool occult blood test

g) Drug-drug interactions

h) Adverse drug reactions (ADRs)

i) Bleeding events defined a priori as major or minor. major bleeding was defined as overt bleeding resulting in transfusion of two or more units of packed blood cells, decrease in hemoglobin level to less than 3 $\mathrm{g} / \mathrm{dL}$, the need for corrective surgery intervention, or the occurrence of intracranial, retroperitoneal, intraocular, body cavity bleeding, or death $(6,13)$.

\section{Statistical analysis}

The extracted data were subjected to statistical analysis using SPSS software (SPSS for windows, version 16, SPSS Inc., Chicago, IL, USA). The entire study population was considered as one group with no subgroup division or analysis.

\section{Results}

A total of 356 patients (177 women and 179 men) between 15-98 years with a mean age of $61.28 \pm 18.98$ were included in this study. The duration of hospital stay ranged from 1 to 88 days, with an average residence time of 11 days. The final outcome of the treatment in patients is demonstrated in figure 1 . As it could be observed the majority of patients were finally discharged after full or partial remission and only less than $2 \%$ of patients died or referred to other centers.

Of the 356 patients included in the study, in 323 patients $(90.73 \%)$ enoxaparin was administered for prophylaxis and the other 33 patients $(9.27 \%)$ received enoxaparin for therapeutic purpose. The frequencies of prophylactic and therapeutic strategies and the indications of enoxaparin in each case are demonstrated in table 1. As it could be observed, the prophylactic indications of enoxaparin were mainly for prevention of coronary artery thrombotic problems and deep vein thrombosis (DVT) in patients undergoing surgery or in immobilized patients. Non-STE-MI was the most common indication for therapeutic use of enoxaparin followed by cerebrovascular accident (CVA).

In majority of the 321 patients $(90.2 \%)$ the indication of enoxaparin was appropriate according to the guidelines of FDA. However, in 8 (2.24\%) patients enoxaparin was prescribed with no indication and in 27 (7.6\%) patients enoxaparin was prescribed in spite of absolute contraindication. These included 4 patients $(14.81 \%)$ with active bleeding, 17 patients (62.96\%) with hypertension and 6 patients $(22.22 \%)$ with subarachnoid or intracranial hemorrhage. In 103 (29\%) patients enoxaparin was prescribed in spite of meeting the criteria for precaution. These include $67(18.8 \%)$ cases of neuraxial anesthesia, 21 (5.9\%) cases of severe hepatic or renal impairment, 9 $(2.5 \%)$ cases of CNS / eye trauma or surgery, 3 (0.8) cases of peptic ulcer and 3 $(0.8 \%)$ patients with retinopathy.

Various dose regiments were used in patients ranging from 20 to $160 \mathrm{mg} /$ day 
Table 1 The prophylactic and therapeutic indications of enoxaparin among the patients

\begin{tabular}{|c|c|c|c|c|}
\hline $\begin{array}{l}\text { Treatment } \\
\text { strategy }\end{array}$ & Prophylactic & Frequency & Therapeutic & frequency \\
\hline \multirow{17}{*}{ Indication } & Surgery & $65(19.9 \%)$ & STE-MI & $3(12 \%)$ \\
\hline & Surgery + Non-STE-MI & $17(5.1 \%)$ & Non-STE-MI & $12(48 \%)$ \\
\hline & Surgery + ACS & $3(0.8 \%)$ & DVT & $2(8 \%)$ \\
\hline & Surgery + hemodialysis & $1(0.3 \%)$ & CVA & \\
\hline & & & & $6(24 \%)$ \\
\hline & ACS & $90(27 \%)$ & VTE in cancer & $1(4 \%)$ \\
\hline & Hemodialysis & $3(0.8 \%)$ & $\begin{array}{l}\text { VTE caused by } \\
\text { implants/ catheters }\end{array}$ & $1(4 \%)$ \\
\hline & Atrial fibrillation & & & \\
\hline & & $8(2.5 \%)$ & & \\
\hline & $\begin{array}{c}\text { Non-STE-MI + unstable } \\
\text { ungina }\end{array}$ & $10(3.1 \%)$ & & \\
\hline & Immobility & $58(17.7 \%)$ & & \\
\hline & Immobility + AF & $3(0.8 \%)$ & & \\
\hline & Immobility + surgery & $28(8.7 \%)$ & & \\
\hline & $\begin{array}{c}\text { Immobility + Non-STE- } \\
\text { MI }\end{array}$ & $4(1.2 \%)$ & & \\
\hline & Immobility + hemodialysis & & & \\
\hline & & $4(1.2 \%)$ & & \\
\hline & No indication & $8(2.24 \%)$ & & \\
\hline
\end{tabular}

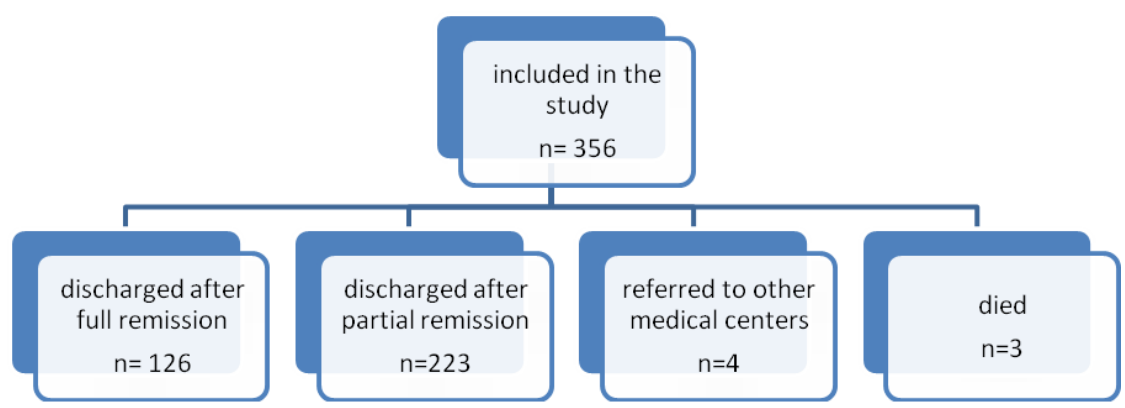

Figure 1 The final outcome of treatment with enoxaparin in the study population 
for prophylaxis and from 40 to 160 mg/Day for therapy. The most frequently administered doses of enoxaparin were $40 \quad \mathrm{mg} /$ Day (administered to 179 patients) and 120 $\mathrm{mg} /$ day (administered to 112 patients) respectively. $186 \quad(52.2 \%)$ patients received appropriate dose of enoxaparin whereas $59(16.6 \%)$ patients were under-dosed and 111 (31.2 \%) were over-dosed. In regard to the duration of treatment, 229 (64.3\%) patients took enoxaparin for the appropriate period, whereas 33 (9.3\%) received the medication for shorter than standard period and 94 (26.4\%) were treated for longer than standard period.

An assessment of the monitoring procedure revealed that the complete blood count (CBC) was only performed on $223(62.6 \%)$ of patients before and during enoxaparin therapy. Measurement of platelet counts was not performed for 31 (8.7\%) and 117 $(32.8 \%)$ patients respectively before and during receiving enoxaparin. In 7 patients $(2.15 \%)$ before prescription, 14 patients $(5.85 \%)$ during treatment and 8 patients $(6.15 \%)$ after treatment platelet counts was less than $100000 \mathrm{~mm}^{3}$. aPTT and INR were not available for $133(37.3 \%)$ and $134(37.6 \%)$ patients before onset of the treatment, respectively and for $277(77.8 \%)$ and $274(76.9 \%)$ patients during treatment, respectively.

The serum potassium levels were not measured for 26 patients $(7.3 \%)$ before initiation of treatment and for 114 patients (32\%) during the treatment.
Among the patients who had been tested for potassium level, in 53\% and $2.2 \%$ of patients potassium level was more than $5 \mathrm{mEq} / \mathrm{l}$ before and during treatment and 6 cases of life threatening hyperkalemia (> $6 \mathrm{mEq} / \mathrm{l}$ ) occurred.

Serum creatinine levels were not controlled before treatment for $25(7 \%)$ patients and during treatment for 97 $(27.24 \%)$ patients. 45 patients (24 female / 21 male) had creatinine clearance of less than $30 \mathrm{ml} / \mathrm{min}$. However, dose adjustment was only performed for 7 patients (15.55\%) and the rest of these patients received more than the required dose. Urine analysis was only performed on $150(42.1 \%)$ of patients during treatment with enoxaparin, of which $22.2 \%$ had hematuria and $19.9 \%$ of patients had normal urine test.

The major adverse effect was thrombocytopenia which occurred in 21 $(5.9 \%)$ of patients. The frequency of other ADRs among the patients was $3.37 \%$ (12 patients) including one patient with injection site bruise and one patient with allergic reactions. No cases of major bleeding were found in the study population. However, hematuria was observed in $58(16.3 \%)$ patients, 23 (39\%) of which were treated with daily doses of $120 \mathrm{mg}$. 31 (53.4\%) of patients who experienced hematuria received concurrent aspirin and clopidogrel and more than half of the patients with hematuria were over 50 years old.

The most important drug-drug interaction in the study population was concurrent use of antiplatelete drugs 
(aspirin and clopidogrel) with enoxaparin which was observed in more than half of the patients (Table 2).

Table 2 Frequencies of drug-drug interactions in patients treated with enoxaparin

\begin{tabular}{cc}
\hline $\begin{array}{c}\text { Co-administered } \\
\text { drug(s) }\end{array}$ & Frequency \\
\hline Aspirin & $48(13.5 \%)$ \\
\hline Clopidogrel & $9(2.5 \%)$ \\
\hline NSAIDs & $58(16.3 \%)$ \\
\hline Warfarin & $3(0.8 \%)$ \\
\hline Aspirin + NSAIDs & $8(2.2 \%)$ \\
\hline Aspirin + & $123(34.6 \%)$ \\
Clopidogrel & \\
\hline Aspirin + & $11(3.1 \%)$ \\
Clopidogrel + & \\
NSAIDs & \\
\hline Aspirin + Warfarin & $2(0.6 \%)$ \\
\hline Aspirin + Clopigrel & $3(0.8 \%)$ \\
+ Warfarin & \\
\hline
\end{tabular}

Other important drug-drug interaction was co-administration of enoxaparin and NSAIDs with or without antiplateletes which accounted for $21.6 \%$ of the total drug interactions. Three patients with DVTand ACS received concurrent warfarin therapy with enoxaparin, while only one patient with DVT had an indication for concurrent warfarin therapy (INR $=1.1$ 1.2). Also two patients with DVT and URI received concurrent enoxaparin, warfarin and aspirin, while only one patient with DVT had an indication for this combinatory regimen (INR $=6.9$ 2.3). Similarly, for three patients with AF, ACS and prosthetic heart valve concurrent enoxaparin, warfarin, aspirin and clopidogrel was prescribed which was not justified (Table 3).

Table 3 Frequencies of irrational use of enoxaparine in patients .

\begin{tabular}{cc}
\hline Irrational use & Frequency: n(\%) \\
\hline Indications & $35(10 \%)$ \\
\hline $\begin{array}{c}\text { Use with } \\
\text { Precaution }\end{array}$ & $103(29 \%)$ \\
\hline Dosage & $170(47.8 \%)$ \\
\hline Duration & $127(35.7 \%)$ \\
\hline Monitoring & $117(33 \%)$ \\
\hline Interaction & $256(72 \%)$ \\
\hline ADR (Hematuria) & $58(16.3 \%)$ \\
\hline
\end{tabular}

\section{Discussion}

In the present study, the DUE of the LMWH "enoxaparin" was evaluated in an educational general hospital during one year. The results demonstrated relatively high rates of inappropriate utilization of enoxaparin in regard to the variables considered in this study namely indication for use, dosing/duration of therapy, drug interaction, and monitoring. What comes next is further discussion of the data retrieved for each variable. 
Indication-contraindication-precaution coronary artery thrombotic problems was found to be the most common prophylactic use of enoxaparin, followed by DVT in patients undergoing surgery or in immobilized patients. Non-STE-MI was the most common indication for therapeutic use of enoxaparin followed by CVA. Enoxaparin was mainly prescribed with appropriate indication. Nevertheless, in about $10 \%$ of the patients inappropriate indication of enoxaparin was observed, of which $7.6 \%$ had contraindication for enoxaparin therapy and $2.24 \%$ had no indication for enoxaparin therapy. Furthermore, in $29 \%$ of patients enoxaparin was prescribed in spite of meeting the criteria for precaution. These findings imply the necessity for more precise consideration of the pertaining factors before allocation of patients to enoxaparin therapy.

\section{Dosage-duration}

Accurate enoxaparin dosing has a significant impact on the thromboembolic disease outcomes and is therefore essential for maintaining a proper balance of efficacy and safety in patients with thromboembolic disease. Inappropriate dosing and/or duration leads to increased risk of bleeding in patients who are over-dosed or treated for longer than standard periods whereas in patients who are underdosed or treated for shorter than standard periods therapeutic outcomes may not be achieved.
Nearly half of the patients $(47.8 \%)$ included in the present study were prescribed inappropriate doses of enoxaparin, the majority of which were over-dosed. By the same way, a large proportion of patients $(35.7 \%)$ were treated with enoxaparin for inappropriate duration, the majority of which received enoxaparin for longer than recommended period. These data are close to what reported in similar studies by Hassani et al. (8) and Fahimi et al. (9). In a study performed by Hassani et al. of the 322 inpatients who were treated with enoxaparin, 53\% were administered inappropriate doses. The majority of patients who received enoxaparin for prophylactic use were over-dosed whereas the patients who were treated with enoxaparin were mainly under-dosed (8). In a similar study carried out by Fahimi et al. on 147 inpatients, a total of $28.7 \%$ of the variables among all subjects were rated as inappropriate for the study setting of which $53.74 \%$ were related to inappropriate dosage or duration of treatment (9).

Considering the similarity of the study setting in terms of regional medical practices in use and nationally adopted therapeutic procedures, it is not of surprise to find quite similar rates for inappropriate enoxaparin dosing regimens in the three mentioned studies which were carried out in Iran. Taking into account the results from all the three studies, the high rate of inappropriate drug utilization for the studied drug (enoxaparin) in Iran necessitates more 
careful consideration and perhaps revision of the drug prescription practices in use.

\section{Monitoring}

Unlike UFH, enoxaparin does not require routine monitoring. Nonetheless, certain laboratory tests such as aPTT (before treatment), CBC, serum potassium level, creatinine clearance, urine analysis, anti Xa factor, stool occult blood test are recommended before commencing and during treatment with enoxaparin (10). Furthermore, since renal excretion is the main elimination route for enoxaparin, dose adjustment is required in patients with renal dysfunction, indicated by creatinine clearance of less than 30 $\mathrm{ml} / \mathrm{min}(6,11)$.

An assessment of the monitoring procedure revealed that anti $\mathrm{Xa}$ factor, and stool occult blood test were not measured for any of the patients included in this study indicating that the physicians did not consider these tests as part of the monitoring procedure. In more than one third of patients, CBC was not tested before and during enoxaparin therapy. By the same way, the platelet counts were not measured for one third (33\%) of patients during enoxaparin therapy. aPTT and INR were not available for more than one third of the patients before onset of the treatment, and for more than 3/4 of patients during treatment. Fahimi et al. (9) also reported lack of appropriate monitoring for $\mathrm{CBC}$ and aPTT during the administration of enoxaparin in $38 \%$ and $24 \%$ of the patients respectively.

The potassium levels were not measured for $32 \%$ of patients during the treatment. in more than half of the patients who were checked for potassium levels, enoxaparin therapy was continued despite hyperkalemia, and 6 cases of life-threatening hyperkalemia was noted in these patients as a result of inappropriate indication of enoxaparin.

Urine analysis was only performed on $42.1 \%$ of patients during treatment with enoxaparin, of which more than half had hematuria. Serum creatinine levels were not controlled for nearly one third of the patients during enoxaparin therapy. Furthermore, of the 45 patients who had creatinine clearance of less than 30 $\mathrm{ml} / \mathrm{min}$, dose adjustment was only performed for 7 patients and the rest of these patients $(10.6 \%$ of the total study population) were over-dosed. This is far more than what reported by Fahimi et al. (9), who reported appropriate enoxaparin dose adjustment in half of the patients who had creatinine clearance less than $30 \mathrm{ml} / \mathrm{min}$.

\section{Drug-drug interaction}

In spite of the potential interactions between enoxaparin and antiplatelete drugs (aspirin and clopidogrel) or NSAIDs which increase the risk of bleeding and should therefore be avoided (12), such combinations were administered to $72 \%$ of the patients. This is far more than what reported in the literature. Few cases of co- 
administered warfarin with wrong indication were also noted among the patients. This rate of drug interaction is far more than what reported by similar studies such as the study by Fahimi et al. (9).

\section{Bleeding}

No cases of major bleeding were found in the study population. However, hematuria was observed in $16.3 \%$ of the patients, which is more than the rate reported by Fahimi et al. (9) but similar to the rate reported by Macie et al. (13).

$40 \%$ of the patients who experienced bleeding received daily doses of 120 mg. which indicates the increased frequency of bleeding in the patients as a consequence of inappropriate dosing. Furthermore, more than half of the patients who had hematuria were treated with a combination of enoxaparin, aspirin and clopidogrel which clearly demonstrates the consequence of this major drug-drug interaction in the study population. The correlation between anti-platelet therapy and bleeding during enoxaparin administration was also demonstrated by Fahimi et al. (9).

Although increased age was not included among the risk factors in this study, it was found that more than half of the patients who experienced bleeding were above 50 years old which suggests an association between increased age and increased risk of bleeding. Macie et al. also reported coadministration of NSAIDs or antiplatelet drugs, increasing patient age, and increasing serum creatinine as the risk factors associated with an increased risk of bleeding. The association between renal dysfunction and increased risk of bleeding was further demonstrated in studies conducted by Khazan et al. (14) and Gilbert et al. (15) who found increased rate of bleeding events in patients with renal failure.

\section{Conclusion}

In conclusion, our results demonstrated inappropriate utilization of enoxaparin in terms of dosage, duration of treatment, precaution/contraindication, drug-drug interaction and monitoring. The consequences of such inappropriate utilization were found to be increased rate of bleeding (hematuria) and few cases of therapeutic failure or death. Hence, there is considerable need for improvement in adherence to the approved guidelines and more careful consideration of both the patient related parameters and the concurrently administered medications before prescription and during treatment with enoxaparin in the studied healthcare setting in order to avoid the unwanted consequences of the inappropriate utilization of enoxaparin. Even more, concordance between the results of the present study and a couple of similar studies carried out in Iran, suggests the necessity for re-consideration and perhaps revision of the current therapeutic procedures and prescription practices in use in the country in an attempt to reduce the rate of inappropriate utilization of enoxaparin 
and avoid its associated medical and economical burden.

Nevertheless, there are potential limitations of this study. First, as a retrospective study, the accuracy of the retrieved data and hence the validity of the results may be affected by inadequate or inaccurate documentation of the relevant data. Second, the study was single-centered which does reduce the generalizability of the results. Hence, larger multi-center studies including larger number of patients in different medical centers are required to further evaluate the DUE of enoxaparin.

\section{Conflict of Interest}

The authors declared no conflict of interest.

\section{References}

1. Sachdeva PD, Patel BG. Drug utilization studies- scope and future perspectives. Int $\mathbf{J}$ Pharm Biol Res 2010;1:11-7.

2. WHO Expert Committee. The Selection of Essential Drugs, Technical Report Series no. 615. Geneva: World Health Organization, 1977.

3. Giancarlo A, Francesco S. Advances in basic, laboratory and clinical aspects of thromboembolic diseases: prevention of venous thromboembolism in high risk patients. Haematologica 1997;82:496-502.

4. Johanne S, Farzin B, Olivier B, Charles Pollack, Marc C, Uwe Z, et al. Efficacy and safety of enoxaparin versus unfractionated heparin during percutaneous coronary intervention: systematic review and metaanalysis. BMJ 2012;344:e553

5. Catia A, Maria A, Pires F, Guilherme BS, Leila B M. Short-Term therapy with enoxaparin or unfractionated heparin for venous thromboembolism in hospitalized patients: utilization study and costminimization analysis. Value Health 2011; 14:89-92.
6. Saheb Sharif-Askari F, Syed Sulaiman SA, Saheb Sharif-Askari N, Al Sayed Hussain A, Railey MJ. Adverse outcomes of anticoagulant use among hospitalized patients with chronic kidney disease: A comparison of the rates of major bleeding events between unfractionated heparin and enoxaparin. Plos One 2014; 9: e106517.

7. Hirsh J, Bauer KA, Donati MB, Gould M, Samama MM, et al. Parenteral anticoagulants american college of chest physicians evidence-based clinical practice guidelines. Chest Journal 2008;133:141-59.

8. Asma H, Khairolla Gh, Azita H, Niaiesh M, Ebrahim $\mathrm{H}$. Enoxaparin utilization evaluation in a cardiovascular teaching hospital. J Urmia Univ of Med Sci 2014;25:241-6.

9. Fahimi F, Baniasadi S, Behzadnia N, Varahram F, Ghazi Tabatabaie L. Enoxaparin Utilization Evaluation: An Observational Prospective Study in Medical Inpatients. Iran J Pharm Res 2008;7:77-82.

10. Haines ST, Racine E, Zeolla M. Venous thromboembolism. In: Dipiro JT, Talbert RL, Yee GC, Matzke GR, Wells BG and Posey LM. (eds.) Pharmacotherapy: A Pathophysiologic Approach. $5^{\text {th }}$ ed. Mc Graw-Hill, New York (2002).

11. Verbeeck R, Musuamba F. Pharmacokinetics and dosage adjustment in patients with renal dysfunction. Eur J Clin Pharmacol 2009;65: 757-73.

12. Tatro D. Drug Interaction Facts. 2010, Walters Kluwer Health; St. Louis.

13. Macie C, Forbes L, Foster G A, Douketis J D. Dosing practices and risk factors for bleeding in patients receiving enoxaparin for the treatment of an acute coronary syndrome Chest 2004; 125:1617-21.

14. Khazan M , Scheuering S, Adamson R, Mathis AS. Prescribing patterns and outcomes of enoxaparin for anticoagulation of atrial fibrillation. Pharmacotherapy 2003; 23:651-8

15. Gilbert K B, Rodgers G M. Utilization and outcomes of enoxaparin treatment for deepvein thrombosis in a tertiary-care hospital. Am J Hematol 2000; 65:285-8. 
